\title{
Reduced ventricular shunt rate in very preterm infants with severe intraventricular hemorrhage: an institutional experience
}

\author{
Clinical article
}

\author{
Nima Alan, B.S., ${ }^{1}$ Sunil Manjila, M.D., ${ }^{1}$ Nori Minich, B.S., ${ }^{3}$ Nancy Bass, M.D., ${ }^{2}$ \\ Alan R. Cohen, M.D., ${ }^{1}$ Michele Walsh, M.D., M.S.c.E., ${ }^{3}$ And Shenandoah Robinson, M.D. ${ }^{1}$
}

Divisions of ${ }^{1}$ Pediatric Neurosurgery, ${ }^{2}$ Pediatric Neurology, and ${ }^{3}$ Neonatology, Rainbow Babies \& Children's Hospital, University Hospitals Case Medical Center Neurological Institute, Case Western Reserve University School of Medicine, Cleveland, Ohio

\begin{abstract}
Object. Although survival for extremely low gestational age newborns (ELGANs) has improved in the past 3 decades, these infants remain prone to complications of prematurity, including intraventricular hemorrhage (IVH). The authors reviewed the outcomes for an entire cohort of ELGANs who suffered severe IVH at their institution during the past 12 years to gain a better understanding of the natural history of IVH and frequency of ventriculoperitoneal (VP) shunt placement in this population.

Methods. Data from the neonatal ICU (NICU) database, neurosurgery operative log, and medical records were used to identify and follow up all ELGANs who suffered a severe IVH between 1997 and 2008. Trends between Period 1 (1997-2001) and Period 2 (2004-2008) were analyzed using the Pearson chi-square test.

Results. Between 1997 and 2008, 1335 ELGANs were admitted to the NICU at the authors' institution within 3 days of birth, and $111(8.3 \%)$ of these infants suffered a severe IVH. Survival to 2 years, incidence of severe IVH, neonatal risk factors (gestational age, birth weight, and incidence of necrotizing enterocolitis), ventriculomegaly on cranial ultrasonography, and use of serial lumbar punctures for symptomatic hydrocephalus were all stable. Infants from Period 2 had a significantly lower incidence of bronchopulmonary dysplasia and sepsis than infants from Period 1 (both $\mathrm{p}<0.001$ ). All ELGANs with severe IVH and ventriculomegaly underwent long-term follow-up to identify shunt status at late follow-up. Twenty-two ELGANs (20\%) with severe IVH required a temporary ventriculosubgaleal (VSG) shunt. Three infants with VSG shunts showed spontaneous hydrocephalus resolution, and 2 infants died of unrelated causes during the neonatal admission. The temporary VSG shunt complication rate was 20\% (12\% infection and 8\% malfunction). Sixteen percent of all ELGANs (18 of 111) with severe IVH eventually required permanent ventricular shunt insertion. Six (35\%) of 17 infants with a permanent VP shunt required at least 1 permanent shunt revision during the 1 st year. The proportion of ELGANs with severe IVH who required a temporary VSG $(35 \%)$ or permanent VP shunt $(30 \%)$ during Period 1 decreased by more than $60 \%$ in Period $2(10 \%$ [p $=0.005]$ and $8.3 \%[\mathrm{p}=0.009]$, respectively).

Conclusions. The authors report for the first time a marked reduction over the past 12 years in the proportion of ELGANs with severe IVH who required surgical intervention for hydrocephalus. Using the NICU database, the authors were able to identify and follow all ELGANs with severe IVH and ventriculomegaly. They speculate that the reduction in ventricular shunt rate results from improved neonatal medical care, including reduced infection, improved bronchopulmonary dysplasia, and postnatal steroid avoidance, which may aid innate repair mechanisms. Multicenter prospective trials and detailed analyses of NICU parameters of neonatal well-being are needed to understand how perinatal factors influence the propensity to require ventricular shunting. (http://thejns.org/doi/abs/10.3171/2012.7.PEDS11504)
\end{abstract}

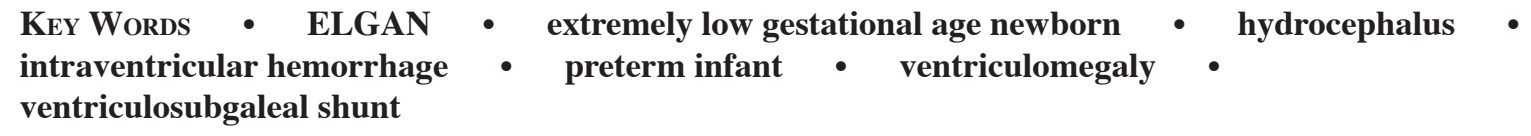

$\mathrm{P}$ RETERM birth remains a challenge in the US. The proportion of infants born preterm $(<37$ weeks gestation) has declined only slightly in recent years despite significant research and preventive efforts, and

Abbreviations used in this paper: $\mathrm{BPD}=$ bronchopulmonary dysplasia; EGA = estimated gestational age; ELGAN = extremely low gestational age newborn; IVH = intraventricular hemorrhage; $\mathrm{LP}=$ lumbar puncture; $\mathrm{NEC}=$ necrotizing enterocolitis; $\mathrm{NICU}=$ neonatal ICU; PVL = periventricular leukomalacia; $\mathrm{VP}=$ ventriculoperitoneal; VSG = ventriculosubgaleal. these infants account for more than $12 \%$ of all live-born infants in the US. ${ }^{9}$ Extremely low gestational age newborns can suffer complications of prematurity, such as $\mathrm{IVH}$, and the risk of this complication increases with lower birth weight and earlier EGA. ${ }^{14}$ Advances in obstetrics and neonatology over the past 3 decades have led to marked improvement in the survival of these vulnerable infants..$^{19}$ Infants who suffer severe IVH are prone to lifelong neurological problems, including cognitive delay, behavioral abnormalities, epilepsy, cerebral palsy, 


\section{N. Alan et al.}

and symptomatic hydrocephalus. ${ }^{8}$ Children with shuntdependent hydrocephalus carry a lifelong burden of the possibility of experiencing a life-threatening ventricular shunt malfunction.

The objective of this study was to retrospectively review the short- and long-term outcomes of ELGANs who were managed in our NICU and who suffered severe IVH and required surgical intervention for symptomatic hydrocephalus over the past decade. The entire cohort of ELGANs with severe IVH and ventriculomegaly underwent long-term follow-up with respect to their need for treatment of symptomatic hydrocephalus. Few studies have examined outcomes of the entire cohort. We hypothesized that the frequency of ventricular shunt placement had declined during the study period. We found that there was a relatively consistent population of preterm infants over the decade, with a stable incidence of severe IVH, ventriculomegaly, and need for serial LPs. However, there was a significant decrease in the proportion of patients requiring temporary or permanent ventricular shunt procedures in Period 2 (2004-2008) compared with Period 1 (19972001) $(p=0.005$ and $p=0.009$, respectively; Pearson chisquare test). This information may provide a framework for informed discussions with parents and caregivers about both the short- and long-term treatment risks and potential outcomes, and may guide research initiatives.

\section{Methods}

Approval for this study was obtained from the University Hospitals Case Medical Center Institutional Review Board. Data from the Neonatal Research Database, the neurosurgical operative log, and medical records at Rainbow Babies \& Children's Hospital were used. The Rainbow NICU database was searched to identify all infants born with a gestational age younger than 30 weeks and a weight less than $1500 \mathrm{~g}$ who were admitted to the hospital within the first 3 days of life between January 1997 and December 2008. To assess trends in the general health of the preterm NICU population, we collected data on birth weight, EGA, survival at 2 years corrected gestational age, incidence of sepsis (defined as a positive blood culture), NEC, and BPD (defined as oxygen dependence at 36 weeks corrected age). Additional information collected from the database for ELGANs included survival to first cranial ultrasonography examination and cranial ultrasonography abnormalities including the most severe IVH grade, PVL, intracranial malformation, and ventricular dilation at any point during the neonatal period and at neonatal discharge. The grade of IVH was based on Papile's criteria. ${ }^{11}$ The grade of IVH, ventricular dilation, and PVL were diagnosed by the attending pediatric radiologist at the time of the study. The most severe IVH grade during the neonatal period was used to stratify the infants. The hospital charts for infants with severe (Grade III and IV) IVH were further reviewed to collect data about whether symptomatic hydrocephalus (defined below) was diagnosed later in life and whether interventions to treat hydrocephalus were performed, including serial LPs, insertion of a temporary or permanent ventricular shunt, and any shunt revisions. For all surviving infants with severe IVH and an ultrasonography diagno- sis of ventriculomegaly who had not undergone shunt surgery prior to neonatal discharge, a minimum of 2 years of follow-up data were obtained from a review of medical charts. None of the infants underwent transfontanel taps, external ventriculostomy, or ventricular endoscopic procedures to treat hydrocephalus.

During the study period, all ELGANs in the Rainbow NICU were routinely followed up using a standard protocol that includes obtaining serial head circumference measurements, neurological examinations, and cranial ultrasonography studies on Days 1-3, Days 7-14, and then, if abnormal, weekly for 4 weeks, and subsequently every 2-4 weeks thereafter. Nearly all of the cohort (97\%) underwent ultrasonography examinations within the first 2 weeks of life. Ventricular dilation occurred in infants with hydrocephalus ex vacuo from encephalomalacia and in infants with symptomatic hydrocephalus. Symptomatic hydrocephalus was defined as clinical signs of increased intracranial pressure, including enlarging head circumference, full anterior fontanel, splayed cranial sutures, decline in neurological examination, poor feeding, lethargy, and irritability accompanied by progressive ventricular dilation noted on serial cranial ultrasonography studies. The pediatric neurosurgery team was consulted if the infant developed any clinical signs of symptomatic hydrocephalus. During the neonatal hospitalization, serial LPs were used as the initial treatment for symptomatic hydrocephalus. The protocol included a trial of LPs to determine whether intermittent CSF removal could effectively control the hydrocephalus. If serial LPs failed to adequately control symptomatic hydrocephalus, as determined by the attending neurosurgeon using the same clinical criteria as above, then a VSG shunt was inserted. During the entire study period (1997-2008), only VSG shunts were used as the initial temporizing surgical procedure. All shunt surgeries in this series of neonates were performed by one of the 2 pediatric neurosurgeons at Rainbow during the study period (A.R.C. and S.R.). To insert a VSG shunt, a ventricular catheter was connected to a low-profile reservoir, and a metal connector was used to attach a valveless $6-\mathrm{cm}$ piece of shunt tubing that was inserted into a subgaleal pocket. Malfunction of a VSG shunt was defined as failure to control symptomatic hydrocephalus and included wound dehiscence or CSF leakage. If the VSG shunt failed while the infant was deemed too small or otherwise at high risk for permanent shunt insertion, then the VSG shunt was removed or revised. If the VSG shunt failed and the infant was a suitable candidate for a permanent shunt, then a VP shunt was inserted. Ideal candidates for a permanent shunt generally weighed more than $2 \mathrm{~kg}$, were without other significant active medical issues, and were infection free. If symptomatic hydrocephalus resolved prior to permanent shunt insertion, then the VSG shunt was not removed. No endoscopic procedures were performed. Former preterm NICU patients underwent follow-up in the Neonatology High-Risk Clinic and had follow-up rates exceeding $90 \%$. All patients with ventriculomegaly and most patients with other neurological sequelae of preterm birth also underwent follow-up in the pediatric neurology and/ or pediatric neurosurgery outpatient clinics. Infants who 
underwent VSG shunt surgery without placement of a permanent ventricular shunt underwent follow-up in the pediatric neurosurgery clinic for the first 2 years of life, and patients with a permanent VP shunt underwent annual follow-up at least during the 1st decade.

Data are presented as the mean \pm SD. Comparisons of nonparametric data were performed using the Pearson chi-square test, and $\mathrm{p}<0.05$ was considered significant. We used SPSS statistical software for the analyses (version 19.0, IBM SPSS, Inc.).

\section{Results}

\section{Overall NICU Population}

Between 1997 and 2008, a total of 1335 ELGANs were admitted to the Rainbow NICU within the first 3 days of life and met this study's inclusion criteria $(<30$ weeks EGA and birth weight $<1500 \mathrm{~g})$. The mean birth weight and EGA did not change significantly between Period 1 (1997-2001, $925 \pm 254 \mathrm{~g}$ and $26.5 \pm 1.8$ weeks) and Period 2 (2004-2008, $916 \pm 265 \mathrm{~g}$ and $26.5 \pm 1.9$ weeks) ( $\mathrm{p}=0.59$ and 0.78 , respectively; 2-tailed t-test) (Fig. 1A). The number of ELGANs who were admitted to the NICU per year also remained relatively constant (mean number of infants/year 111, range 95-121 infants/ year) (Fig. 1B), which suggests a stable regional referral pattern to this institution over the study period. The Rainbow NICU admits newborns from across northern Ohio. During the study period, the proportion of very low birth weight infants $(<1500 \mathrm{~g})$ and the proportion of very preterm infants $(<32$ weeks EGA) in Ohio, and in Cleveland, the major metropolitan area in northern Ohio, closely mimicked the national trends (Fig. 1C). Infant survival, defined as the proportion of ELGANs who survived to 2 years of age (mean $84.6 \%$, range $81.1 \%-88.4 \%$ ), and incidence of NEC also remained relatively constant from 1997 to 2008 (Fig. 2 and Table 1). By contrast, the incidence of 2 other common complications in ELGANs, sepsis and BPD, decreased from Period 1 (1997-2001) to Period 2 (2004-2008) (both p < 0.001, Pearson chisquare test) (Table 1 and Fig. 2).

Of 1335 ELGANs born between 1997 and 2008, 1295 survived to undergo an initial cranial ultrasonography study within the first 3 days of life, and $111(8.6 \%)$ suffered a severe (Grade III or IV) IVH. The proportion of infants

TABLE 1: Incidence of neonatal complications (1997-2008)

\begin{tabular}{lccr}
\hline & \multicolumn{2}{c}{$\%$} & \\
\cline { 2 - 3 } \multicolumn{1}{c}{ Parameter } & $\begin{array}{c}\text { Period 1 } \\
(1997-2001)\end{array}$ & $\begin{array}{c}\text { Period 2 } \\
(2004-2008)\end{array}$ & p Value* \\
\hline neonatal mortality & 14.7 & 15.5 & 0.710 \\
BPD & 41.0 & 25.1 & $<0.001$ \\
NEC & 9.0 & 10.6 & 0.377 \\
sepsis & 35.6 & 24.6 & $<0.001$ \\
severe (Grade III/IV) IVH & 7.0 & 9.4 & 0.149 \\
\hline
\end{tabular}

* Determined using the Pearson chi-square test. Values in boldface are significant.

\section{Rainbow Preterm Infant Population}

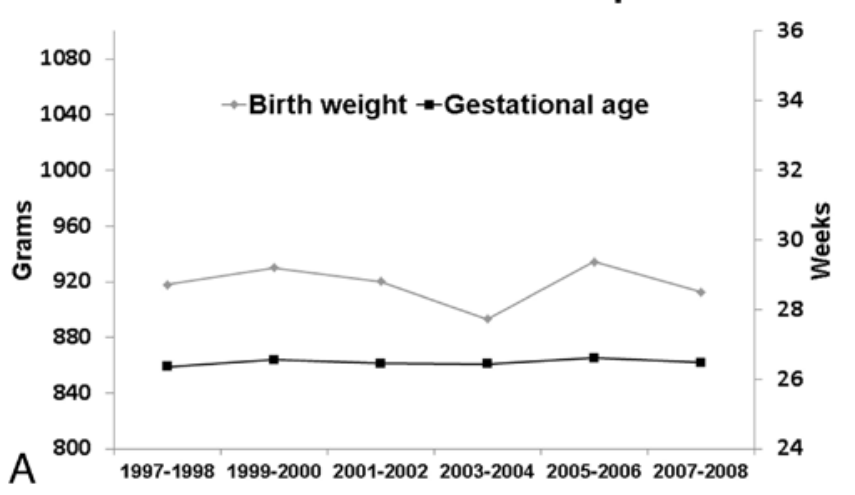

Very Preterm Infant Survival

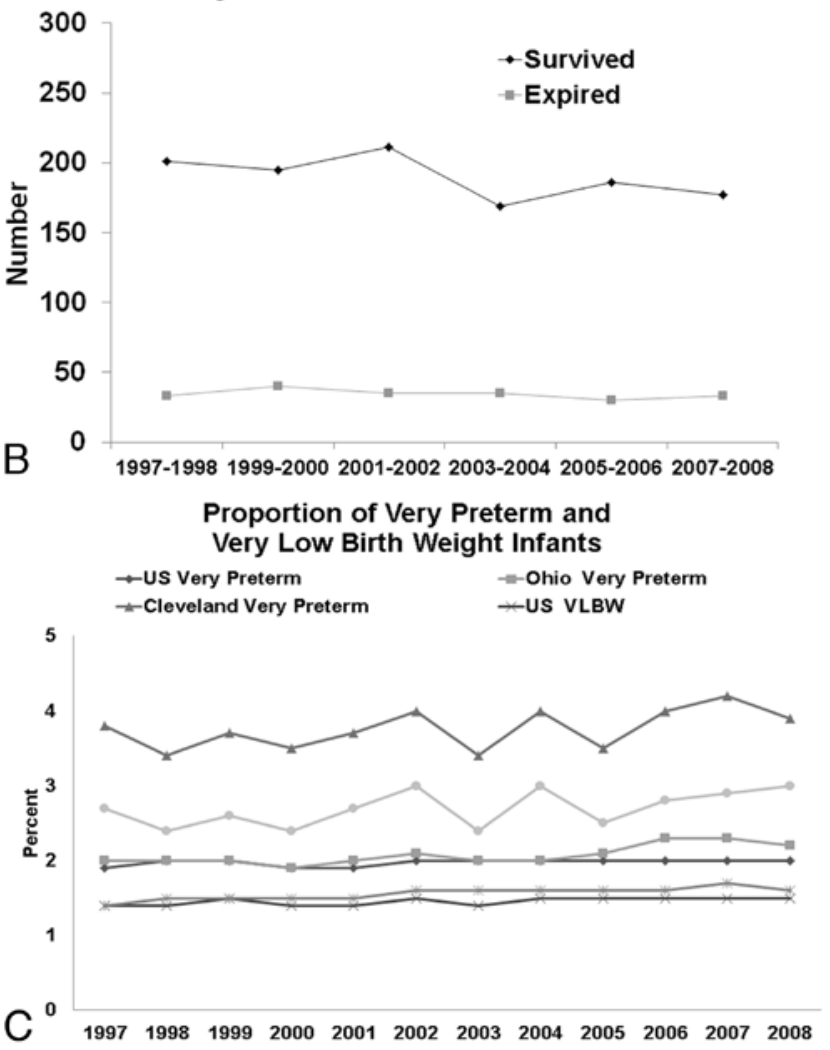

FIG. 1. A: Graph depicting the EGA and birth weight for all infants admitted to the Rainbow NICU within 3 days of birth between 1997 and 2008. There was no significant change in the EGA or birth weight over the study period. Given that the risk of severe IVH correlates highly with younger EGA and lower birth weight, the stable EGA and birth weight over the 12 years suggests that the risk of the overall NICU population did not change significantly over the study period. B: Graph showing the number of very preterm infants who were admitted to the Rainbow NICU within 3 days of birth between 1997 and 2008 and met the inclusion criteria ( $<1500 \mathrm{~g}$ and $<30$ weeks) for this study. The number was relatively stable over the study period. C: Graph depicting the percentage of live births born very preterm ( $<32$ weeks EGA) or at very low birth weight (VLBW) $(<1500 \mathrm{~g})$ in the US, Ohio, and Cleveland, the major metropolitan area in northern Ohio (marchofdimes.com/peristats, accessed May 1, 2012). 


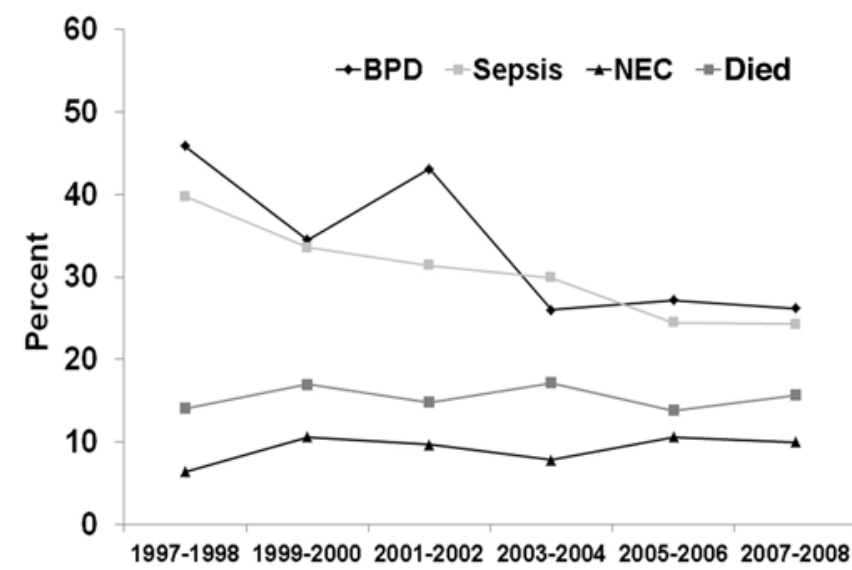

FIG. 2. Graph showing that the proportion of very preterm infants who died and the proportion diagnosed with NEC remained relatively consistent over the decade. The incidence of BPD and sepsis decreased from Period 1 (1997-2001) to Period 2 (2004-2008) (both $p<$ 0.001). Also see Table 1.

who survived to undergo initial cranial ultrasonography (mean 97.1\%/year, range 95.4\%-99.1\%/year) remained stable from 1997 to 2008. The proportion of ELGANs who developed an IVH of Grade I/II (mean 17.3\%, range $12.7 \%-33.3 \%$ ) or Grade III/IV (mean $8.6 \%$, range $4.4 \%$ $12.9 \%$ ) over the study period was also relatively stable (Fig. 3 ). Of the 111 infants who suffered a severe IVH during 1997-2008, 27.0\% were also diagnosed using cranial ultrasonography as having PVL. The incidence of PVL remained stable over the period of study (Fig. 3).

Ventricular dilation on cranial ultrasonography was detected in infants with hydrocephalus ex vacuo and in infants with symptomatic hydrocephalus. Among infants with any grade of IVH from 1997 to 2008, 22.1\% (74 of 335 infants) had ventricular dilation reported on at least 1 cranial ultrasonography study obtained at some point during the neonatal hospitalization. Not surprisingly, only $1.4 \%$ of infants with a Grade I/II IVH had ventricular dilation, while $59.5 \%$ of infants (66 of 111) with severe IVH showed ventricular dilation at some point in their neonatal course. The proportion of infants with severe (Grade III/IV) IVH who were diagnosed with ventricular

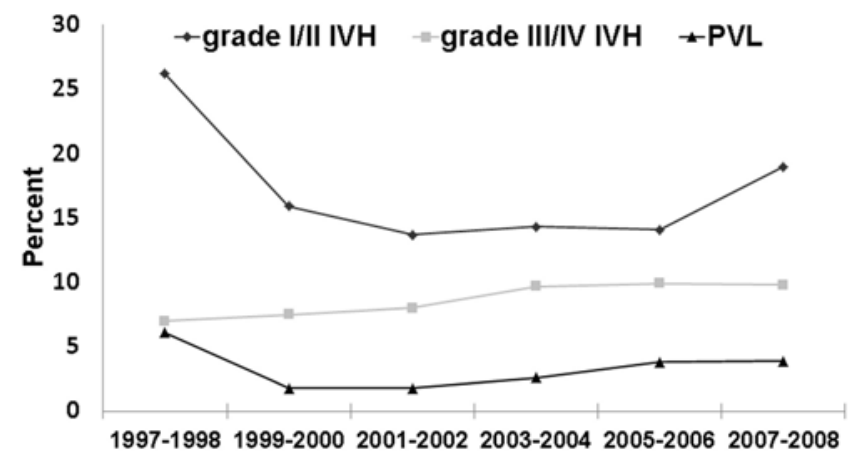

FIG. 3. Graph showing the proportion of infants who suffered a Grade I/II or Grade III/IV IVH, or PVL detected on cranial ultrasonography during 1997-2008. The most severe IVH at any point in the neonatal period was used to stratify infants with multiple grades of IVH. The incidence of these lesions did not change significantly over the study period. dilation by ultrasonography remained stable from 1997 to 2008 (mean 5.1\%, range 2.7\%-7.8\%) (Fig. 4). Ten patients with severe IVH who survived the neonatal admission had unresolved ventricular dilation and no shunt at the time of discharge from the neonatal hospitalization. Nine of those patients had extended follow-up (mean 9.4 years, median 9 years; 1 lost to follow-up), and only 1 subsequently developed symptomatic hydrocephalus and required a permanent shunt insertion at 7 months.

\section{Symptomatic Hydrocephalus Treatment}

Serial LPs were the initial treatment for symptomatic hydrocephalus. The only data consistently available were whether an infant underwent a trial of serial LPs. Details of the LPs, such as how many were performed and how much CSF was removed each time, were not consistently available from the medical charts for analysis. The proportion of ELGANs with severe IVH and at least transient symptomatic hydrocephalus requiring serial LPs decreased by one-third from Period 1 (52\% [21 of 40]) to Period 2 (35\% [17 of 48]), but this was not significant $(\mathrm{p}=0.107$, Pearson chi-square test) (Fig. 4). The proportion of all ELGANs who subsequently developed symptomatic hydrocephalus due to severe IVH and required serial LPs during the neonatal hospitalization remained stable between the periods (Period 1, 3.7\% [21 of 571]; and Period 2, 3.3\% [17 of 510]). Forty-one percent of ELGANs (19 of 46) with severe IVH and symptomatic hydrocephalus treated with serial LPs did not require any surgical intervention.

Shunt data were reviewed from January 1997 through December 2010. Of the 111 ELGANs who suffered a severe IVH, 22 (19.8\%) underwent insertion of a temporary VSG shunt during the neonatal admission. A trial of serial LPs had failed in all of these infants. No VSG shunts were inserted after discharge from the neonatal admission. The mean birth weight for infants who were treated with a VSG

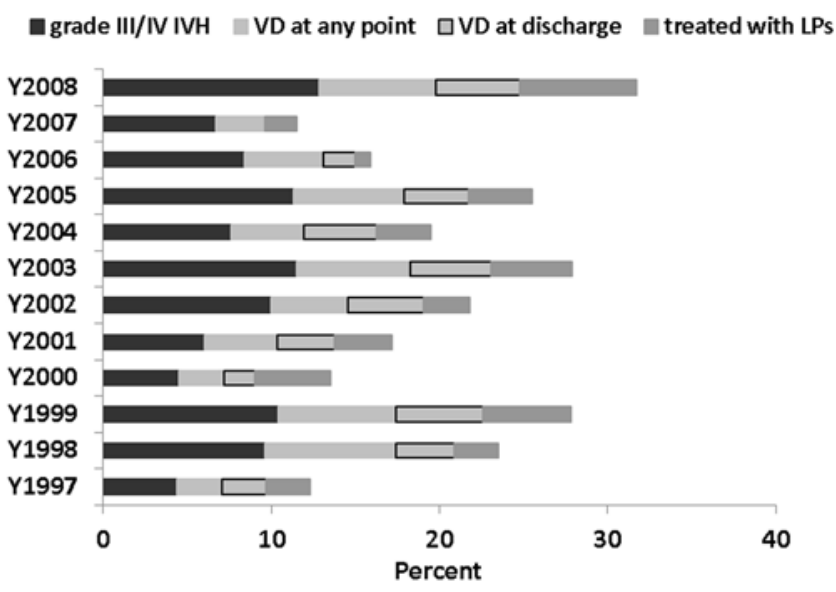

FIG. 4. In this study ventriculomegaly was diagnosed by cranial ultrasonography and included both hydrocephalus ex vacuo and symptomatic hydrocephalus with symptoms and signs of increased intracranial pressure. The graph shows the proportion of very preterm infants with severe IVH, those with severe IVH plus ventricular dilation (VD) at any point during the neonatal admission, the proportion with severe IVH and persistent ventricular dilation at neonatal discharge, and the proportion treated with serial LPs. There was no significant change during the study period. 
shunt was $985 \mathrm{~g}$, the mean birth EGA was 26.6 weeks, and the mean age at VSG shunt insertion was 5.1 weeks (median 4 weeks, range 2-14 weeks). By comparison, the mean birth weight of ELGANs with severe IVH who did not undergo any shunt surgery was lower (823 g). Twenty patients (90.9\%) who underwent VSG shunt insertion survived the neonatal admission. Three of those who survived (15\%) had spontaneous resolution of hydrocephalus after temporary VSG shunt placement and did not require a permanent shunt at any point (mean follow-up 7 years, range 3.2-10.2 years). One infant with severe IVH and ventricular dilation who had been discharged as a neonate had late presentation of symptomatic hydrocephalus at 7 months and underwent permanent shunt insertion. Thus, $18(16.2 \%)$ of 111 very preterm infants who suffered a severe IVH underwent insertion of a permanent VP shunt during infancy. Of the 18 patients who required insertion of a permanent shunt, $12(66.7 \%)$ underwent insertion during the neonatal admission. Six patients underwent shunt insertion after they were discharged from the hospital as neonates, and 5 underwent insertion before 6 months of age. Overall, the mean age at the time of permanent shunt insertion was 3.2 months (median 3 months). For infants who had a permanent shunt inserted after discharge as a neonate, the mean age at the time of insertion was 5.2 months (range 3-7 months).

Despite a relatively stable incidence of severe IVH, the proportion of ELGANs with severe IVH who required a temporary or permanent shunt decreased over the 12 years (Fig. 5). Comparison of the proportion of infants with severe IVH who required a temporary shunt during Period 1 (mean $35.0 \%$ ) with those during Period 2 (mean $10.4 \%$ ) showed a $70 \%$ decline in the proportion of infants who required surgical treatment for symptomatic hydrocephalus ( $\mathrm{p}=0.005$, Pearson chi-square test). Similarly, the requirement for a permanent shunt decreased from $30 \%$ during Period 1 to $8.3 \%$ during Period 2 ( $\mathrm{p}=0.009$ ).

\section{Shunt Complications}

None of the infants suffered a life-threatening complication related to the VSG shunt insertion, and surgical mortality was zero. Two $(9.1 \%)$ of the 22 very preterm infants with severe IVH who underwent VSG shunt insertion died as neonates of conditions unrelated to hydrocephalus. One infant born in 1999 with a birth weight of $792 \mathrm{~g}$ died at 61 days (1 month after VSG insertion) of numerous complications of prematurity, including status epilepticus and pulmonary complications. An infant born in 2000 with a birth weight of $740 \mathrm{~g}$ died at 133 days of complications of NEC, approximately 2 months after VSG shunt insertion. The VSG had malfunctioned with a proximal occlusion, and the hydrocephalus was managed using serial LPs because the infant was deemed too ill to undergo a revision. A third infant born in 2001 with a birth weight of $653 \mathrm{~g}$ who underwent VSG shunt insertion at 3.5 months and permanent VP shunt insertion at 5 months had no revisions prior to his death at 17 months due to pulmonary complications. No ELGAN with severe IVH born after 2001 who underwent surgical treatment of hydrocephalus died. Although this is a small series, the mortality from any cause for infants who underwent surgical treatment of hydrocephalus remained low over the study period.

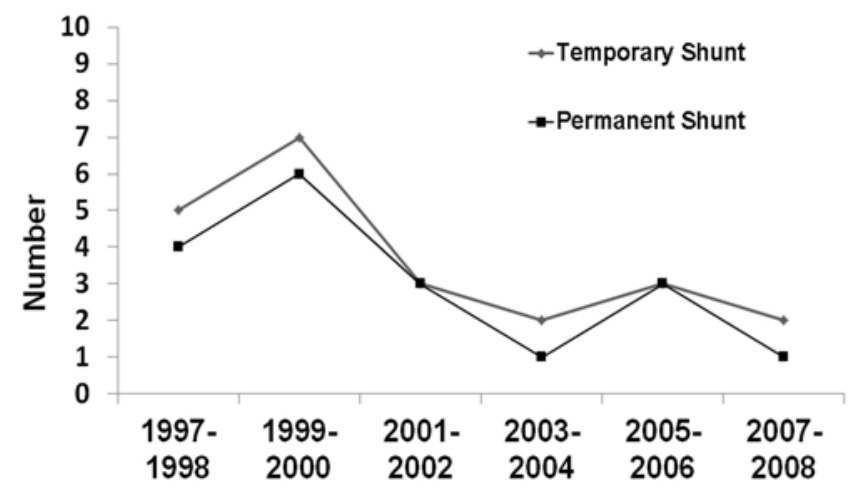

FIG. 5. Graph showing the number of temporary VSG shunts and the number of VSG shunts converted to a permanent VP shunt per year from 1997 to 2008. A comparison of shunt insertion rate between Period 1 (1997-2001) and the more recent Period 2 (2004-2008) shows a significant reduction in the number of temporary VSG shunts and permanent VP shunts inserted $(p=0.005$ and $p=0.009$, respectively; Pearson chi-square test).

Five of 22 patients who underwent VSG shunt insertion suffered a wound complication, infection, or a malfunction of the initial VSG shunt $(22.7 \%$ per patient, $25 \%$ per long-term survivor, and $20 \%$ per procedure). Removal of the VSG shunt due to wound dehiscence was required in 2 patients, and these patients were presumed to have infection. One of those patients had spontaneous resolution of the hydrocephalus, and the other had replacement of the VSG shunt 2 weeks later, followed by eventual permanent shunt insertion. Another patient had a Staphylococcus aureus infection that was successfully treated with intravenous antibiotics. A VSG shunt revision due to proximal occlusion was required in 1 patient. The VSG shunt malfunctioned in another patient who was too ill to undergo a revision (discussed above). Thus, in this series the infection rate for VSG shunts was $13.6 \%$ per patient ( 3 of 22), and $12 \%$ per VSG shunt procedure (3 of 25), and the VSG shunt malfunction rate was $9.1 \%$ per patient and $8 \%$ per procedure.

All 18 patients who underwent permanent shunt insertion survived the 1st year of life. One patient moved out of state, and the long-term shunt revision history is unknown. Follow-up was available for the remaining 17 patients with permanent shunts (94\%). Six (35.3\%) of the 17 patients required at least 1 revision of the permanent shunt during the 1st year of life. Four suffered shunt malfunctions, and 2 suffered a shunt infection. One patient who never underwent a revision died at 17 months of pulmonary issues, and 16 patients survived long term (mean follow-up 6.9 years, median 7 years). Of those 16 infants who underwent permanent shunt insertion and for whom long-term follow-up is available, $6(38 \%)$ have never undergone a shunt revision, with a mean follow-up of 8.5 years (median 9.4 years). Nearly two-thirds (10 of 16) of the long-term survivors have not had any revisions since the 1st year of life, including 4 patients who underwent revisions during the 1st year. This suggests that revisions during the 1st year do not necessarily predispose patients to a higher rate of revisions later in childhood. The mean number of revisions after the 1 st year of life was 0.7 per 


\section{N. Alan et al.}

patient. Only one of the revisions after 1 year of age was complicated by infection. Overall, the mean number of revisions was 0.3 per patient-year of follow-up. None of the patients in this series underwent endoscopic fenestration of complex loculated hydrocephalus. Of the 16 long-term survivors with a permanent shunt, the majority also suffered from spasticity, epilepsy, cognitive delay, and behavioral abnormalities such as attention deficit disorder, hyperactivity, and autism spectrum disorders. All received special education services for cognition or behavioral deficits, therapy services for motor and speech delay, or both.

\section{Discussion}

This retrospective single-institution analysis of an entire recent cohort of ELGANs is unique in that it monitored all infants with severe IVH and ventricular dilation or shunt surgery at neonatal discharge for an extended period of follow-up. We found that over the past 12 years the overall population of ELGANs had a relatively stable incidence of neonatal risk factors, such as birth weight and gestational age and other complications of prematurity, except for the incidence of BPD and sepsis, which declined. The incidence of severe IVH also remained relatively constant. This clinical pattern is similar to that in some recent reports of outcomes during the past decade for very preterm infants, ${ }^{14,16}$ but not in others. ${ }^{6}$ In this study, a relatively high proportion $(55.4 \%)$ of ELGANs with severe IVH had ventricular dilation noted on at least 1 cranial ultrasonography examination performed at some point during the neonatal hospitalization. In this series, however, ventricular dilation included both hydrocephalus ex vacuo and symptomatic hydrocephalus associated with increased intracranial pressure, which may explain why the number of patients with ventricular dilation is higher than that reported by others who only included ventricular dilation that required surgical treatment. ${ }^{6}$

\section{Reduction in the Need for Surgical Intervention}

In the setting of a relatively consistent population of very preterm infants with severe IVH, we report for the first time a decrease in the proportion of these infants who required surgical intervention for symptomatic hydrocephalus. We found a significant decline in the placement of temporary VSG shunts from Period 1 (1997-2001) to Period 2 (2004-2008, $p=0.005)$. In the recent study from St. Louis Children's Hospital, the number of temporary VSG shunts inserted increased over a similar study interval. ${ }^{6}$ In that study, the mean annual percentage of infants (EGA $<40$ weeks and birth weight $<1500$ g) with severe IVH who required a temporary shunt was $30.8 \%,{ }^{6}$ while ours over the same interval (1997-2008) was $19.8 \%$. We also found a significant decline in the need for permanent VP shunt insertion between Periods 1 and $2(\mathrm{p}=0.009)$. This reduction in the need for ventricular shunting is important because of the lifelong burden of shunt-dependent hydrocephalus and potential shunt malfunction. In the US during 2008 (the most recent year for which data are available), approximately 61,000 infants were born weighing less than $1500 \mathrm{~g} .{ }^{9}$ If the outcomes at our insti- tution are extrapolated to the larger US population, then approximately 5490 ELGANs (9\%) per year will suffer a severe IVH. Moreover, approximately $460(0.8 \%)$ of these infants per year will require insertion of a permanent ventricular shunt that may require lifelong surveillance and treatment.

The reason for this unprecedented reduction in the need for surgical intervention is not yet clear. The percentage of preterm infants born in Rainbow's catchment area across northern Ohio parallels national trends. Through examination of the NICU survival data, we found relatively constant survival curves over the past 12 years for short-term survival (survival to initial cranial ultrasonography within 3 days of birth) and survival to 2 years. This suggests that the decline in the need for surgical intervention was not due to lack of survival of sick infants with severe IVH. Also, the proportion of infants with severe IVH, ventricular dilation, and symptomatic hydrocephalus requiring treatment with serial LPs remained relatively constant over the study period, which suggests that the incidence of infants who potentially would require surgical intervention was stable. We were unable to collect sufficient detail about the LPs, except to know whether a trial of LPs was attempted in each patient, which limited the conclusions we could draw about the efficacy of serial LPs. The neonatologists, pediatric radiologists, neurologists, and neurosurgeons responsible for the care of these infants did not change over the study period, which suggests that the reduction is not due to a change in practitioners or practice philosophy. Our threshold for surgical intervention in preterm infants with severe IVH is similar to that of others. ${ }^{3}$

We endorse the concept that prolonged symptomatic hydrocephalus can potentially impair neurodevelopment, and we tended to intervene surgically within 2 days if treatment with serial LPs failed in the infant. Most of the patients with severe IVH and ventricular dilation on cranial ultrasonography at neonatal discharge $(90 \%$ follow-up) or with temporary neonatal surgical intervention (100\% follow-up) underwent long-term follow-up to confirm that no child suffered from under-treatment of symptomatic hydrocephalus. To summarize, the decline in the need for shunting was not due to the following factors: 1) a lower risk population of preterm infants being admitted to the NICU; 2) lack of treatment of the sickest infants; 3) change in the proportion of infants who developed a severe IVH, ventricular dilation, or need for serial LPs; 4) change in the treating physicians and surgeons or treatment philosophy for symptomatic hydrocephalus; or 5) lack of follow-up.

We propose that the subgroup of ELGANs with severe IVH who require surgical intervention for symptomatic hydrocephalus composes a sicker cohort than infants who do not develop persistent symptomatic hydrocephalus. The infants who require a permanent ventricular shunt tend to have more cognitive impairment and a greater burden of other chronic neurological problems than infants with severe IVH who do not require shunts. ${ }^{8}$ One explanation for the observed reduction in the need for surgical intervention may be that advances in neonatology practice may be supporting the overall health of 


\section{Reduction of ventricular shunting in preterm infants}

these very fragile infants better, minimizing additional CNS injury, and increasing the chances of spontaneous brain recovery and resolution of the hydrocephalus. In support of this concept, there has also been a reduction in the incidence of severe cystic PVL over the past decade. ${ }^{17}$ Changes in neonatology practice over the past decade include greater use of caffeine for apnea and erythropoietin for anemia of prematurity in some centers. Both of these interventions have been shown to enhance neurodevelopmental outcomes in a few studies, ${ }^{2,10,13}$ but the efficacy has not been demonstrated yet in rigorous Phase III trials. Nutrition practices for these infants have also changed over the past 12 years with an increased awareness of the benefits of feeding with human milk. ${ }^{4,12}$ Revised resuscitation measures, decreased use of mechanical ventilation, and postnatal corticosteroids may contribute to the improved outcomes. ${ }^{1,18}$ Finally, infection reduction practices have been implemented in the past several years, such as the strict adherence to sterile technique for bedside procedures. Together, these changes may be minimizing the degree of illness and secondary injury for these infants. These changes also may have not been well detected by the parameters routinely collected for the NICU database. For example, in the NICU database, sepsis is recorded as a positive blood culture. The impact for an infant who experiences a relatively asymptomatic course from a more benign microbe and early effective antibiotic treatment is likely quite different from an infant with multisystem organ failure from florid gram-negative sepsis. Our current data collection methods do not capture these differences. Serum biomarkers may provide an additional means to classify and track the severity of illness in these infants.

Another possible explanation for the decrease in the need for ventricular shunting is that some infants are genetically more predisposed to poor outcomes from severe IVH or other CNS insults due to preterm birth. A recent study revealed that children who were born preterm and developed cerebral palsy had an excessive peripheral leukocyte response to an inflammatory challenge at 5 years of age that was not present in children who were similarly born preterm but were neurologically normal. ${ }^{7}$ The Rainbow NICU serves a diverse population, but the demographic pattern is relatively stable. This is a single-institution study and the study population may differ from that of other NICUs.

Overall in this series, temporary VSG shunts were inserted in $19.8 \%$ of 111 ELGANs with severe IVH, and $16.2 \%$ of the 111 infants eventually required insertion of a permanent VP shunt. Two infants died in the neonatal period, and 3 had spontaneous resolution of symptomatic hydrocephalus. Thus, $77.3 \%$ of the temporary shunts (17 of 22) were converted to permanent shunts, a proportion similar to what other authors have reported. ${ }^{6}$

Our rate of ventricular shunt complication is similar to that which has been previously reported.5,15 This is a very vulnerable population with high surgical risks, including poor wound healing, anemia and thrombocytopenia, poor nutrition, and numerous other medical problems. The infection rate was $12 \%$ per temporary shunt procedure. Of note, we classified any VSG shunt associated with a wound dehiscence as infected, regardless of whether a positive CSF culture was obtained. Protocols used in older children at the time of shunt surgery that have been shown to reduce the risk of shunt infection, including preoperative baths and skin preparation with chlorhexidine and a perioperative course of nasal mupirocin, are not currently approved for use at our institution for infants younger than 2 months corrected age.

The risk of VSG shunt malfunction in this series was $8 \%$ per procedure, which is similar to that in other recent series. ${ }^{6}$ Most catheter occlusions were likely due to obstruction by debris from the resolving IVH clot. For temporary ventricular shunting, we prefer to use VSG shunts rather than ventricular access devices (ventricular reservoirs). No study has yet shown a distinct advantage of VSG shunts over ventricular access devices. ${ }^{6}$

In this study, combining the NICU data set, neurosurgical operative log, and medical records allowed us to document the natural history of the entire population of ELGANs who suffered a severe IVH during the past 12 years. We were able to follow the entire cohort over the long term because of the consistent surveillance provided by the High Risk Neonatology Clinic. A limitation of this study is that it is a single-institution retrospective experience. Because it is a retrospective analysis, not all of the data desired, such as details regarding the uniformity and efficacy of serial LPs, were available. As a single-institution study, the findings from this report may not be widely applicable to other centers with different populations due to genetic and environmental factors.

\section{Conclusions}

To our knowledge, this is the first report of a significant reduction in the need for temporary and permanent ventricular shunting for symptomatic hydrocephalus in ELGANs with severe IVH. This reduction is clinically significant because a permanent VP shunt is associated with a lifelong risk of a potentially life-threatening shunt malfunction. The reduction in the need for surgical intervention for symptomatic hydrocephalus occurred in the setting of a relatively stable incidence of neonatal risk factors, incidence of severe IVH, ventricular dilation, need for serial LPs, and practice management pattern. We were able to identify and track the entire NICU population of ELGANs who suffered IVH and were at risk for developing symptomatic hydrocephalus during childhood. To better understand the predictive factors driving the decreased need for surgical intervention after severe IVH in very preterm infants, a multiinstitutional study that collects detailed information about the overall NICU course is needed.

\section{Disclosure}

The authors report no conflict of interest concerning the materials or methods used in this study or the findings specified in this paper.

Author contributions to the study and manuscript preparation include the following. Conception and design: Robinson, Manjila, Bass, Cohen, Walsh. Acquisition of data: Robinson, Alan, Walsh. Analysis and interpretation of data: Robinson, Manjila, Minich, Bass, Cohen, Walsh. Drafting the article: Robinson, Alan, Manjila, 


\section{N. Alan et al.}

Minich. Critically revising the article: all authors. Reviewed submitted version of manuscript: all authors. Approved the final version of the manuscript on behalf of all authors: Robinson. Statistical analysis: Robinson, Alan, Minich, Walsh. Administrative/technical/ material support: Robinson, Walsh. Study supervision: Robinson, Bass, Walsh.

\section{Acknowledgments}

The authors would like to thank their colleagues in pediatric anesthesia, neonatology, pediatric neurology, and pediatric radiology for their contributions to the care of these patients.

\section{References}

1. Aly H, Hammad T, Essers J, Wung J: Is mechanical ventilation associated with intraventricular hemorrhage in preterm infants? Brain Dev 34:201-205, 2012

2. Brown MS, Eichorst D, Lala-Black B, Gonzalez R: Higher cumulative doses of erythropoietin and developmental outcomes in preterm infants. Pediatrics 124:e681-e687, 2009

3. de Vries LS, Liem KD, van Dijk K, Smit BJ, Sie L, Rademaker $\mathrm{KJ}$, et al: Early versus late treatment of posthaemorrhagic ventricular dilatation: results of a retrospective study from five neonatal intensive care units in The Netherlands. Acta Paediatr 91:212-217, 2002

4. Ehrenkranz RA, Das A, Wrage LA, Poindexter BB, Higgins $\mathrm{RD}$, Stoll BJ, et al: Early nutrition mediates the influence of severity of illness on extremely LBW infants. Pediatr Res 69: 522-529, 2011

5. Fulkerson DH, Vachhrajani S, Bohnstedt BN, Patel NB, Patel AJ, Fox BD, et al: Analysis of the risk of shunt failure or infection related to cerebrospinal fluid cell count, protein level, and glucose levels in low-birth-weight premature infants with posthemorrhagic hydrocephalus. Clinical article. J Neurosurg Pediatr 7:147-151, 2011

6. Limbrick DD Jr, Mathur A, Johnston JM, Munro R, Sagar J, Inder $\mathrm{T}$, et al: Neurosurgical treatment of progressive posthemorrhagic ventricular dilation in preterm infants: a 10-year single-institution study. Clinical article. J Neurosurg Pediatr 6:224-230, 2010

7. Lin CY, Chang YC, Wang ST, Lee TY, Lin CF, Huang CC: Altered inflammatory responses in preterm children with cerebral palsy. Ann Neurol 68:204-212, 2010

8. Maitre NL, Marshall DD, Price WA, Slaughter JC, O'Shea TM, Maxfield C, et al: Neurodevelopmental outcome of in- fants with unilateral or bilateral periventricular hemorrhagic infarction. Pediatrics 124:e1153-e1160, 2009

9. Mathews TJ, Miniño AM, Osterman MJ, Strobino DM, Guyer B: Annual summary of vital statistics: 2008. Pediatrics 127: 146-157, 2011

10. Neubauer AP, Voss W, Wachtendorf M, Jungmann T: Erythropoietin improves neurodevelopmental outcome of extremely preterm infants. Ann Neurol 67:657-666, 2010

11. Papile LA, Burstein J, Burstein R, Koffler H: Incidence and evolution of subependymal and intraventricular hemorrhage: a study of infants with birth weights less than 1,500 gm. J Pediatr 92:529-534, 1978

12. Schanler RJ: Outcomes of human milk-fed premature infants. Semin Perinatol 35:29-33, 2011

13. Schmidt B, Roberts RS, Davis P, Doyle LW, Barrington KJ, Ohlsson A, et al: Long-term effects of caffeine therapy for apnea of prematurity. N Engl J Med 357:1893-1902, 2007

14. Stoll BJ, Hansen NI, Bell EF, Shankaran S, Laptook AR, Walsh $\mathrm{MC}$, et al: Neonatal outcomes of extremely preterm infants from the NICHD Neonatal Research Network. Pediatrics 126:443-456, 2010

15. Taylor AG, Peter JC: Advantages of delayed VP shunting in post-haemorrhagic hydrocephalus seen in low-birth-weight infants. Childs Nerv Syst 17:328-333, 2001

16. Vassilyadi M, Tataryn Z, Shamji MF, Ventureyra EC: Functional outcomes among premature infants with intraventricular hemorrhage. Pediatr Neurosurg 45:247-255, 2009

17. Volpe JJ: Brain injury in premature infants: a complex amalgam of destructive and developmental disturbances. Lancet Neurol 8:110-124, 2009

18. Watterberg KL: Policy statement-postnatal corticosteroids to prevent or treat bronchopulmonary dysplasia. Pediatrics 126:800-808, 2010

19. Wilson-Costello D, Friedman H, Minich N, Siner B, Taylor G, Schluchter M, et al: Improved neurodevelopmental outcomes for extremely low birth weight infants in 2000-2002. Pediatrics 119:37-45, 2007

Manuscript submitted November 13, 2011

Accepted July 25, 2012.

Please include this information when citing this paper: published online August 31, 2012; DOI: 10.3171/2012.7.PEDS11504.

Address correspondence to: Shenandoah Robinson, M.D., Department of Neurosurgery, Boston Children's Hospital, Hunnewell 2, 300 Longwood Avenue, Boston, Massachusetts 02115. email: Shenandoah.robinson@childrens.harvard.edu. 